\title{
Solução analítica para um modelo de dispersão de poluentes com reação fotoquímica na camada limite atmosférica
}

\author{
Analytical solution for a pollutant dispersion model with photochemical reaction \\ in the atmospheric boundary layer
}

Guilherme Jahnecke Weymar ${ }^{1}$, Bardo Ernst Josef Bodmann², Daniela Buske ${ }^{3}$, Jonas da Costa Carvalho ${ }^{4}$ e Marco Túllio Vilhena ${ }^{5}$

${ }^{1}$ Doutorando, PROMEC, UFRGS, Porto Alegre, RS, Brasil

${ }^{2}$ Doutor em Física, PROMEC, UFRGS, Porto Alegre, RS, Brasil

${ }^{3}$ Doutora em Engenharia Mecânica, IFM, UFPel, Pelotas, RS, Brasil

${ }^{4}$ Doutor em Meteorologia, Faculdade de Meteorologia, UFPel, Pelotas, RS, Brasil

${ }^{5}$ Doutor em Engenharia Mecânica, PROMEC, UFRGS, Porto Alegre, RS, Brasil

\begin{abstract}
Resumo
Este trabalho apresenta uma solução analítica para a equação de advecção-difusão tridimensional aplicada à dispersão de poluentes reativos lançados na Camada Limite Atmosférica (CLA). Algumas substâncias quando emitidas na CLA sofrem reações fotoquímicas, sendo assim um termo fonte é incluído na equação de advecção-difusão para representar esta reação. Os campos de concentração do poluente obtidos pela solução proposta são comparados com dados de razão de mistura obtidos pelo monitoramento da qualidade do ar na Região Metropolitana de Porto Alegre. Com a análise dos resultados pode-se verificar que a inserção do termo proposto para representar uma reação fotoquímica de um poluente reativo consiste numa nova proposta para a previsão de concentração desse tipo de poluente na CLA.
\end{abstract}

Palavras-chave: equação advecção-difusão, reação fotoquímica, solução analítica, método GILTT.

\begin{abstract}
This paper presents an analytical solution for three-dimensional advection-diffusion equation applied to the dispersion of reactive pollutants emitted into the Atmospheric Boundary Layer (PBL). Some substances when emitted in PBL suffer photochemical reactions, so a source term is included in the advection-diffusion equation to represent this reaction, turning the model more realistic. The pollutant concentration fields obtained by the proposed solution are compared with the mixing ratio data obtained by monitoring the air quality in the metropolitan region of Porto Alegre. With the analysis of the results can be seen that the inclusion of the term proposed to represent a photochemical reaction of a reactive pollutant, a is a new proposal for the prediction of pollutant concentration in PBL.
\end{abstract}

Keywords: advection-diffusion equation, photochemical reaction, analytical solution, GILTT method. 


\section{Introdução}

O interesse em preservar a qualidade do ar vem crescendo consideravelmente nos últimos anos, devido ao aumento da emissão de poluentes na atmosfera provocado pelo crescimento do desenvolvimento industrial e a queima de combustíveis fósseis por veículos automotores. Assim, para o controle da qualidade do ar necessita-se de um instrumento interpretativo, como um modelo matemático capaz de ligar a causa (fonte de poluição) ao efeito (concentração do poluente). A utilização de determinados modelos matemáticos em adição às medidas produz um salto de qualidade na gestão da poluição atmosférica, pois os modelos permitem uma descrição mais completa do desenvolvimento do fenômeno de transporte, de sua distribuição espacial em pontos onde não há medidas e da previsão do campo de concentração (Moreira e Tirabassi, 2004).

Nos dias de hoje, pode-se dizer que um dos maiores problemas causados pela poluição atmosférica em áreas urbanas são provocados pelos oxidantes fotoquímicos, esses são formados na atmosfera através da reação entre compostos orgânicos voláteis (COV's) e óxidos de nitrogênio $\left(N O_{x}\right)$ em presença de luz solar, sendo o principal deles o ozônio $\left(\mathrm{O}_{3}\right)$. Portanto, o poluente em interesse neste trabalho é o ozônio, considerado um poluente secundário, é um gás azulado, reativo e cerca de 1,6 vezes mais pesado que o oxigênio. $O$ caráter oxidante deste gás pode ocasionar diversos danos à fauna e a flora. Além disso, o $\mathrm{O}_{3}$ contribui para o efeito estufa uma vez que o composto apresenta uma banda de absorção em 9,6 $\mu \mathrm{m}$, na qual absorve radiação terrestre (Colbeck e Mackenzie, 1994).

Segundo (Athinson-Palombo et al., 2006) os automóveis são as principais fontes de emissão dos percursores de ozônio. Mesmo sabendo da complexidade da química atmosférica, quando a atmosfera tem predominância de compostos nitrogenados a formação do ozônio troposférico é bem conhecida. Contudo, quando há presença de radicais hidroxila $(\mathrm{OH})$ e hidrocarbonetos estes causam um desequilíbrio atmosférico, resultando no aumento da formação de ozônio.

(Finlayson-Pitts e Pitts, 1997) afirma que a principal reação na produção do ozônio na atmosfera é entre o oxigênio atômico e o molecular:

$$
\mathrm{O}+\mathrm{O}_{2}+\mathrm{M} \longrightarrow \mathrm{O}_{3}+\mathrm{M}
$$

onde $M$ é um terceiro elemento, tal como $\mathrm{N}_{2}$ ou $\mathrm{O}_{2}$, que remove a energia da reação e estabiliza o $\mathrm{O}_{3}$. A altitudes mais baixas (troposfera) onde há apenas radiação com comprimentos de onda maiores que $280 \mathrm{~nm}$, a única fonte de oxigênio atômico é a fotodissociação do dióxido de nitrogênio:

$$
\mathrm{NO}_{2}+h v \longrightarrow \mathrm{NO}+\mathrm{O}
$$

Onde o fóton $h v$ tem comprimento de onda entre 290 e $430 \mathrm{~nm}$. Considerando a presença de radicais hidroxila $(\mathrm{OH})$ e hidrocarbonetos, em particular, radicais peróxi ( $R \mathrm{O}_{2} *$, onde $\mathrm{R}$ é um grupo alquil) produzidos na oxidação de moléculas de hidrocarbonetos e que reagem com o $\mathrm{NO}$ para formar o $\mathrm{NO}_{2}$, resultando no aumento da formação de ozônio, como a reação a seguir:

$$
\mathrm{RO}_{2} *+\mathrm{NO} \longrightarrow \mathrm{NO}_{2}+\mathrm{RO} *
$$

estabelecendo assim um ciclo onde o processo líquido das reações (2), (1) e (3) é:

$$
\mathrm{RO}_{2} *+\mathrm{O}_{2}+h v \longrightarrow \mathrm{RO} *+\mathrm{O}_{3}
$$

Neste trabalho apresentamos uma solução analítica para a equação de advecção-difusão tridimensional aplicada à dispersão de poluentes formados a partir de uma reação fotoquímica na Camada Limite Atmosférica (CLA), a resolução do problema é feita com o uso das técnicas da Transformada de Laplace e GILTT (Generalized Integral Laplace Transform Technique) (Buske et al., 2012), para isso considera-se que o poluente seja volátil e que sua formação se dê através das reações (2), (3) e (1) respectivamente.

\section{Metodologia}

Inicialmente analisamos a produção de ozônio na troposfera através das reações (2), (3) e (1). A fotodissociação do dióxido de nitrogênio (reação 2), pode ser descrita (Finlayson-Pitts e Pitts, 2000) como um processo de primeira ordem, representado por:

$$
\frac{\mathrm{d}\left[\mathrm{C}_{\mathrm{NO}_{2}}\right]}{\mathrm{d} t}=-J_{\mathrm{NO}_{2}}\left[C_{\mathrm{NO}_{2}}\right]
$$

onde o fóton deve ter comprimento entre $290 \mathrm{~nm}<\lambda<$ $430 \mathrm{~nm}$, a frequência de fotólise de $\mathrm{NO}_{2}$ representada pelo coeficiente $J_{\mathrm{NO}_{2}}$ pode ser calculada (Jacobson, 2005) da seguinte maneira:

$$
J_{\mathrm{NO}_{2}}=\int_{0}^{\infty} \phi(\lambda) \sigma(\lambda) F(\lambda) \mathrm{d} \lambda
$$

onde $F(\lambda)$ é o fluxo actínio integrado esfericamente, $\sigma(\lambda)$ seção de choque de absorção e $\phi(\lambda)$ rendimento quântico para a fotodissociação. Neste trabalho como um primeiro estudo, o coeficiente $J_{\mathrm{NO}_{2}}$ é considerado constante.

Para modelar o campo de concentração de ozônio será utilizada a equação de advecção-difusão tridimensional transiente acrescentada de um termo de reação fotoquímica (processo de primeira ordem), este termo representa a fotodissociação do elemento $\mathrm{NO}_{2}$ em $\mathrm{O}_{3}$ :

$$
\frac{\partial \bar{c}}{\partial t}+\vec{v} \cdot \nabla \bar{c}=\nabla \cdot(\mathbb{K} \cdot \nabla) \bar{c}+J_{N_{2}} C_{N_{2}}
$$


sendo $\bar{c}$ a concentração média do poluente (ozônio), $\vec{v}=$ $(\bar{u}, \bar{v}, \bar{w})$ a velocidade média do vento, $\mathbb{K}$ a matriz dos coeficientes de difusão $\mathbb{K}=\operatorname{diag}\left(K_{x}, K_{y}, K_{z}\right)$. O domínio de interesse é um cubo com dimensões $L_{x}, L_{y}$ e $h$, na qual $h$ é a altura da camada limite planetária. A equação (7) está sujeita as condições de contorno de fluxo nulo nas faces do cubo, concentração inicial nula (em $t=0)$ e condição de fonte $\bar{c}(0, y, z, t)=0$ observa-se que a produção do ozônio acontece apenas pela reação fotoquímica.

Para resolver o problema proposto, aplica-se o método espectral na variável $y$, ou seja, expande-se a concentração de poluente em uma série em termos das autofunções do problema de Sturm-Liouville associado e aplica-se o operador integral, $\int_{0}^{L_{y}}(\cdot) Y_{n}(y) \mathrm{d} y$ transformando assim a equação (7) em um sistema de equações advectivas-difusivas bidimensionais transientes, e com as seguintes hipóteses simplificativas: A advecção é dominante na direção do eixo- $x\left[\bar{u} \frac{\partial \bar{c}}{\partial x}>>\frac{\partial}{\partial x}\left(K_{x} \frac{\partial \bar{c}}{\partial x}\right)\right]$; a direção do vento é orientada no eixo- $x[\vec{v}=(\bar{u}, 0,0)] \mathrm{e}$ o coeficiente de difusividade turbulenta lateral, $K_{y}=$ $K_{y}(z)$, obtém-se a seguinte equação:

$$
\begin{gathered}
\frac{\partial \bar{c}_{m}(x, z, t)}{\partial t}+\bar{u} \frac{\partial \bar{c}_{m}(x, z, t)}{\partial x}=K_{z} \frac{\partial^{2} \bar{c}_{m}(x, z, t)}{\partial z^{2}}+K_{z}^{\prime} \frac{\partial \bar{c}_{m}(x, z, t)}{\partial z} \\
-\beta_{m}^{2} K_{y} \bar{c}_{m}(x, z, t)+J_{\mathrm{NO}_{2}} C_{\mathrm{NO}_{2}} \int_{0}^{L_{y}} Y_{n}(y) \mathrm{d} y
\end{gathered}
$$

a equação (8) é resolvida pela técnica GILTT. Para tanto, aplica-se a transformada de Laplace na variável temporal resultando num problema estacionário bidimensional (Wortmann et al., 2005):

$$
\begin{aligned}
& \bar{u} \frac{\partial \bar{C}_{m}(x, z, r)}{\partial x}=K_{z} \frac{\partial^{2} \bar{C}_{m}(x, z, r)}{\partial z^{2}}+K_{z}^{\prime} \frac{\partial \bar{C}_{m}(x, z, r)}{\partial z} \\
& -\left(K_{y} \beta_{m}^{2}+r\right) \bar{C}_{m}(x, z, r)+J_{N_{2}} \bar{C}_{N_{2}} \int_{0}^{L_{y}} Y_{n}(y) \mathrm{d} y
\end{aligned}
$$

Aplicando novamente o método espectral, agora na variável $z$, obtém-se uma equação diferencial ordinária de primeira ordem:

$$
B Y^{\prime}(x, r)+E Y(x, r)=H(r)
$$

onde $Y(x, r)$ é o vetor das componentes $\bar{C}_{m, l}(x, r)$, e $B=$ $\left(b_{l, j}\right), E=\left(e_{l, j}\right)$ e $H=\left(h_{l}\right)$ as matrizes cujas entradas, respectivamente, são:

$$
\begin{gathered}
b_{l, j}=\int_{0}^{h} \bar{u} \psi_{l}(z) \psi_{j}(z) \mathrm{d} z \\
e_{l, j}=\alpha_{l}^{2} \int_{0}^{h} K_{z} \psi_{l}(z) \psi_{j}(z) \mathrm{d} z-\int_{0}^{h} K_{z}^{\prime} \psi_{l}^{\prime}(z) \psi_{j}(z) \mathrm{d} z+ \\
\int_{0}^{h}\left(K_{y} \beta_{m}^{2}+r\right) \psi_{l}(z) \psi_{j}(z) \mathrm{d} z
\end{gathered}
$$

$$
h_{l}=J_{\mathrm{NO}_{2}} \bar{C}_{\mathrm{NO}_{2}} \int_{0}^{L_{y}} Y_{n}(y) \mathrm{d} y \int_{0}^{h} \psi_{l}(z) \mathrm{d} z
$$

Cabe salientar que quando aplicado os operadores integrais estes foram normalizados, deste modo a equação diferencial ordinária (10) é resolvida analiticamente pela técnica da transformada de Laplace e diagonalização, obtendo-se $Y(x, r)=\bar{C}_{m, l}(x, r)$ e que portanto $\bar{C}_{m}(x, z, r)$ está bem determinada. Para obter $\bar{c}_{m}(x, z, t)$ aplica-se a transformada inversa de Laplace em $\bar{C}_{m}(x, z, r)$, a inversão é feita numericamente. Uma vez que $\bar{c}_{m}(x, z, t)$ é conhecida, a solução final da equação de advecçãodifusão (7) é dada pela equação:

$$
\bar{c}(x, y, z, t)=\sum_{m=0}^{M} \bar{c}_{m}(x, z, t) Y_{m}(y)
$$

\section{Resultados e parametrizações}

Para uma utilização correta dos modelos de transporte e difusão na atmosfera não pode prescindir de um estudo sobre suas capacidades de representarem corretamente situações reais. Quando posível, recomenda-se verificar a confiabilidade do modelo utilizado com os dados, com os cenários topográficos e meteorológicos típicos da área a ser analisada (Moreira et al., 2007).

Com o objetivo de investigar o comportamento das concentrações do ozônio $\left(\mathrm{O}_{3}\right)$, o modelo foi comparado com as médias das concentrações observadas durante os dias no mês de janeiro do ano de 2006 na estação de Esteio, os dados utilizados foram fornecidos pela estação da Fundação Estadual de Proteção Ambiental Henrique Luiz Roessler (FEPAM). A localização da Estação de monitoramento em Esteio é apresentada na Figura (1).

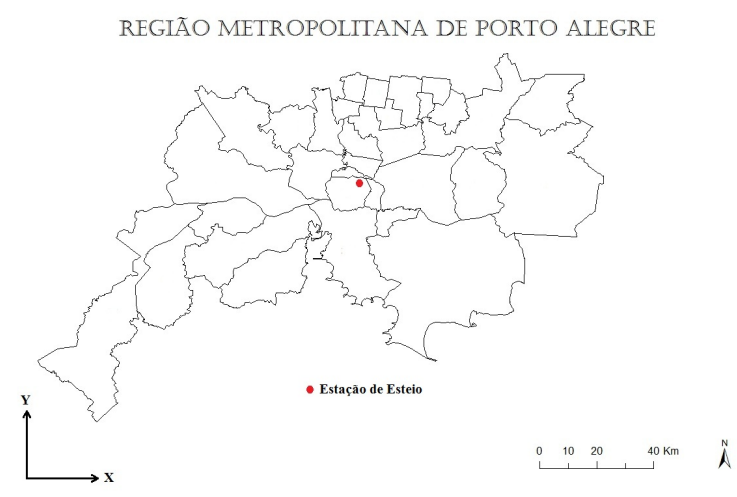

Figura 1: Localização da estação de monitoramento em Esteio (FEPAM)

Neste trabalho foram escolhidas as seguintes parametrizações de turbulência, para calcular as concentrações no sistema tridimensional: 


\subsection{Parametrizações turbulentas e Perfil do Vento}

Como um exemplo de aplicação para a solução proposta utilizou-se os seguintes coeficientes de difusão vertical e lateral sugeridos por Degrazia et al. (1997), derivados de Batchelor (1949), para condições convectivas:

$$
\begin{gathered}
K_{z}=0,22 w_{*} h\left(\frac{z}{h}\right)^{\frac{1}{3}}\left(1-\frac{z}{h}\right)^{\frac{1}{3}}\left[1-e^{\frac{-4 z}{h}}-0,0003 e^{\frac{8 z}{h}}\right] \\
K_{y}=\frac{\sqrt{\pi} \sigma_{v}}{16\left(f_{m}\right)_{v} q_{v}}
\end{gathered}
$$

em que $\sigma_{v}$ é o desvio padrão Euleriano da velocidade turbulenta longitudinal dado por:

$$
\sigma_{v}^{2}=\frac{0,98 c_{v}}{\left(f_{m}\right)_{v}^{\frac{2}{3}}}\left(\frac{\psi_{\epsilon}}{q_{v}}\right)^{\frac{2}{3}}\left(\frac{z}{h}\right)^{\frac{2}{3}} w_{*}^{2}
$$

onde $\left(f_{m}\right)_{v}=0,16$ é a componente vertical da frequência normalizada do pico espectral, $q_{v}=4,16 \frac{z}{h}$ é a função estabilidade, $\psi_{\epsilon}$ é a função taxa de dissipação molecular expressada como (Hojstrup, 1982) (Caughey, 1982):

$$
\psi_{\epsilon}^{1 / 3}=\left[\left(1-\frac{z}{h}\right)^{2}\left(\frac{z}{-L}\right)^{-2 / 3}+0,75\right]^{1 / 2}
$$

Para descrever o campo de vento para a simulação da dispersão de poluentes, a equação utilizada para a parametrização do vento é descrita por uma lei de potência expressa pela seguinte equação (Panofsky e Dutton, 1984):

$$
\frac{\bar{u}}{\overline{\bar{u}}_{1}}=\left(\frac{z}{z_{1}}\right)^{\alpha}
$$

na qual $\bar{u}$ e $\bar{u}_{1}$ são as velocidades médias horizontais do vento nas alturas $z$ e $z_{1}$, e $\alpha$ é um expoente que está relacionado com a intensidade da turbulência (Irwin, 1979).

\subsection{Resultados Numéricos}

Pode-se observar na Figura (2) que as comparações entre as concentrações do $\mathrm{O}_{3}$ simulado $\left(C_{p}\right)$ e observado $\left(C_{o}\right)$ revelaram que o modelo simulou bem a evolução dos valores observados no período das $11 \mathrm{hs}$ às $19 \mathrm{hs}$ para os dias do mês de janeiro de 2006, mesmo considerando o coeficiente $J_{\mathrm{NO}_{2}}$ como uma constante, não dependendo assim do fluxo actínio integrado esfericamente $(F(\lambda))$, da seção de choque de absorção $(\sigma(\lambda))$ e do rendimento quântico da fotodissociação $(\phi(\lambda))$.

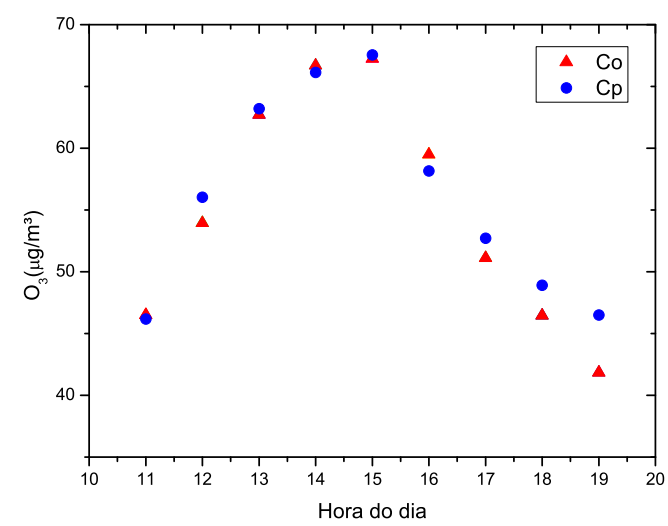

Figura 2: Comparação entre as médias das concentrações horárias durante os dias do mês de janeiro simuladas e observadas para estação de Esteio (FEPAM)

\section{Conclusão}

Neste trabalho apresentamos uma nova representação analítica para a solução da equação de advecção-difusão (com um pequeno erro na inversão numérica da transformada inversa de Laplace) para o problema de dispersão de um poluente, e que o modelo em questão considera a reação fotoquímica de um poluente primário lançado na atmosfera. As soluções analíticas são de fundamental importância para entender e descrever fenômenos físicos, pois elas levam em conta explicitamente todos os parâmetros envolvidos no problema, de modo que suas influências podem ser confiavelmente investigadas. Mesmo com as aproximações apresentadas neste trabalho, o modelo conseguiu reproduzir de modo aceitável o comportamento da concentração de ozônio durante o período das $11 \mathrm{hs}$ às $19 \mathrm{hs}$.

\section{Referências}

Athinson-Palombo, C., Miller, J. A., Balling, J., Robert, C. (2006). Quantifuing the ozone "weeken effect"at various locations in phoenix, arizona. Atmospheric Environment, (40), 7644-7658.

Batchelor, G. K. (1949). Diffusion in a field of homogeneous turbulence, Eulerian analysis, Australian Journal of Scientific Research 2, pp. 437-450.

Buske, D., Vilhena, M. T., Tirabassi, T., Bodmann, B. (2012). Air pollution steady-state advection-diffusion equation: the general three-dimensional solution. Journal of Envir Protec, 4, 1-10.

Caughey, S. J. (1982). Diffusion in the convective boundary layer, Atmospheric Turbulence and Air Pollution Mo- 
delling, Editted by F.T.M. Nieuwstadt and H. Van Dop, Reidel, Boston.

Colbeck, I., Mackenzie, A. (1994). Air pollution by photochemical oxidants, vol 1. Elsevier Science.

Degrazia, G. A., Velho, H. F. C., Carvalho, J. C. (1997). Nonlocal exchange coefficients for the convective boundary layer derived from spectral properties. Contributions to Atmospheric Physics, pp. 57-64.

Finlayson-Pitts, B. J., Pitts, J. N. (1997). Tropospheric air pollution ozone, airborne toxics, plycyclic aromatic hydrocarbons and particles. Science, 276, 1045-1051.

Finlayson-Pitts, B. J., Pitts, J. N. (2000). Spectroscopy and photochemistry fundamentals. Science, pp. 43-85.

Hojstrup, J. (1982). Velocity spectra in the unstable boundary layer. Journal of Amospheric Sciences, 39, 2239-2248.

Irwin, J. S. (1979). A theorical variation of the wind profile power-low exponent as a function of surface roughness and stability. Atmospheric Environment, 13, 191-194.

Jacobson, M. Z. (2005). Fundamentals of Atmospheric Modeling, $2^{\circ}$ edn. Cambridge University Press.

Moreira, D., Tirabassi, T. (2004). Modelo matemático de dispersão de poluentes na atmosfera: um instrumento técnico para gestão ambiental. Ambiente $\mathcal{E}$ Sociedade, 7, 169-171.

Moreira, D. M., Vilhena, M. T., Carvalho, C. (2007). Tritium dispersion simulation in the atmosphere from angra i nuclear power plant. International Journal of Nuclear Energy Science and Technology, 3, 118-130.

Panofsky, H. A., Dutton, J. A. (1984). Atmospheric Turbulence. John Wiley \& Sons, New York.

Wortmann, S., Vilhena, M. T., Moreira, D., Buske, D. (2005). A new analytical approach to simulate the pollutant dispersion in the pbl. Atmospheric Environment, 39, 2171-2178. 